Published by Al-Nahrain College of Medicine P-ISSN 1681-6579

E-ISSN 2224-4719

Email: iraqijms@colmed-alnahrain.edu.iq

http://www.colmed-alnahrain.edu.iq

http://www.iraqijms.net

Iraqi JMS 2020; Vol. 18(1)

\title{
Screening of Silent Myocardial Ischemia in Type 2 Diabetic Patients with Additional Atherogenic Risk Factors; The Applicability of Exercise Stress Test
}

\author{
Zeki Y. Al-Ethary ${ }^{1}$ MSC, Moayed B. Alrikabi ${ }^{2}$ FICMS \\ ${ }^{1}$ Coronary Care Unit, Al-Imamein Al-Kadhimein Medical City, Baghdad, Iraq, ${ }^{2}$ Dept. of Medicine, College of Medicine, \\ Al-Nahrain University, Baghdad, Iraq
}

\begin{abstract}
Background Silent myocardial ischemia (SMI) is a major clinical entity. SMI is highly prevalent and occurs more commonly in patients with diabetes. It is a predictor of mortality.

Objective To assess SMIs in type 2 diabetic patients with atherogenic risk factors using exercise stress test (EST) and to evaluate its applicability as a screening test.

Methods The study was conducted on sixty-three type 2 diabetic symptomless patients (37 male, 26 female patients), and without resting electrocardiographic (ECG) signs of ischemia. Patients were divided into 2 groups; Group I: patients with one or none of the atherogenic risk factors and Group II: patients with 2 or more of the atherogenic risk factors or peripheral vascular disease (PVD) alone. EST was carried out for all patients. Ischemia was diagnosed by ECG. Other tests were carried out as echocardiography, ultrasound Doppler of carotid arteries.

Results Only 57/63 (90.5\%) patients had a diagnostic EST; 11 (19.3\%) tested to be positive and 46 (80.7\%) tested to be negative. ECG indicated ischemic features in 10 patients with positive EST. Ultrasound Doppler showed PVD in 3 out of 41 patients. Using multivariate analysis, there was a significant association of positive EST with PVD, hyperlipidemia, smoking, and hypertension.

Conclusion As the number of the atherogenic risk factors in diabetic patients increase, the incidence of SMI increases. Therefore, EST is applicable and practicable for screening of SMI in type 2 diabetic patients with 2 or more atherogenic risk factors.

Keywords

Citation Silent Myocardial ischemia, Exercise Stress test, Diabetes mellitus.

Al-Ethary ZY, Alrikabi MB. Screening of silent myocardial ischemia in type 2 diabetic patients with additional atherogenic risk factors; the applicability of exercise stress test. Iraqi JMS. 2020; 18(1): 70-78. doi: 10.22578/IJMS.18.1.10
\end{abstract}

List of abbreviations: $C A D=$ Coronary artery disease, $E C G=$ Electrocardiography, EST = Exercise stress test, IHD = Ischemic heart disease, $\mathrm{OHA}=$ Oral hypoglycemic agents, $\mathrm{PVD}=$ Peripheral vascular disease, $\mathrm{SMI}=$ Silent myocardial infarction

\section{Introduction}

I schemic heart disease (IHD) is the most common, serious, chronic, life-threatening illness in developed countries. Diabetes mellitus (DM) accelerates coronary and peripheral atherosclerosis and is frequently associated with dyslipidemia and increases the risk of angina, myocardial infarction, and sudden coronary death. Asymptomatic or silent myocardial ischemia (SMI) is more frequent in diabetic than in nondiabetic patients ${ }^{(1)}$.

Diabetes is a common disease with prevalence rates that are predicted to grow significantly over the next several decades (2). Its complications affect many organ systems, especially through vascular changes 
(atherosclerotic changes) and are responsible for the majority of morbidity and mortality associated with the disease.

SMI screening is particularly necessary for those patients who show additional risk factors other than diabetes ${ }^{(3)}$. The exercise stress test (EST) is the low-cost, non-invasive screening test used most widely for SMI (4).

This study aimed to assess SMIs in type 2 diabetic patients with atherogenic risk factors using EST and to evaluate its applicability as a screening test.

\section{Methods}

A cross sectional single center study was conducted on 63 type 2 diabetic patients attending the Diabetic Clinic in Al-Imamein AlKadhimein Medical City from November 2004 to May 2005. All patients had a previous diagnosis of type 2 diabetes mellitus. The diagnosis was based on WHO criteria and under supervision of an internal medicine specialist.

Diabetic patients with any of the following criteria were excluded: typical angina or chest pain; signs of myocardial ischemia during resting echocardiography (ECG); severe and poor prognosis systemic diseases (e.g., liver failure or chronic renal failure); age $>70$ years; claudication observed at $<400 \mathrm{~m}$; and left bundle branch block on resting ECG.

The study was conducted according to the principles of declaration of Helsinki. Patients were divided into 2 groups; Group I: patients with one or none of the atherogenic risk factors and Group II: patients with 2 or more of the atherogenic risk factors or peripheral vascular disease (PVD) alone.

All patients were recruited and underwent the EST to detect silent myocardial ischemia in relation to the presence or absence of additional atherogenic risk factors for coronary artery disease (CAD). The patients were informed about the benefits and the risks of the test with full explanation of the test procedure by which they have signed a written consent. If the patient was on $\beta$-blocker or a calcium-channel blocker, the EST was postponed in order to stop these drugs gradually and substituted by an alternative one (e.g., captopril). EST was done with abstinence of these drugs for at least 48 hours. Prior to the EST, the following measures were done: resting ECG; measurement of blood pressure (BP), pulse rate, HbA1c, Lipid profile (total cholesterol, triglyceride, high density lipoprotein (HDL), low density lipoprotein (LDL), and very low density lipoprotein (VLDL)), fundoscopy, ultrasound doppler of carotid arteries except some patients. Echocardiography was done for all patients as soon as possible (1/4 - 1 hour) after the EST.

Treadmill exercise test (CASE 16 exercise testing system, Marquette Medical System, Inc.1998, Milwaukee, Wisconsin, USA) according to Bruce protocol was carried out for all patients participating in this study. Each stage lasts for 3 minutes. At the end of each stage and recovery period, which lasts for 3-5 minutes, a 12-lead ECG record was printed and BP measurement was recorded. The EST was halted when one of the following endpoints was reached:

1) Target heart rate, $85 \%$ of the anticipated heart rate (220 beats/minus age in yrs);

2) Extreme fatigue;

3) A reduction of the systolic blood pressure;

4) Hypertensive response (plus systolic blood pressure $>230 \mathrm{mmHg}$ and/or blood pressure diastolic $>120 \mathrm{mmHg}$ );

5) Arrhythmias which are life threatening;

6) Severe depression of ST segment $>3 \mathrm{~mm}$; Acute elevation of ST segment.

7) Desire of the patient.

Echocardiography (Diagnostic Ultrasound Scanner, Combison 530D, kretz-Technik, Inc.1997, Zipf, Austria) was carried out for all patients after the EST. It was done to detect the ischemic changes like hypokinesia, dyskinesia, poor left ventricular function, and impaired diastolic relaxation.

Fundoscopy (Topcon, Optical CO., LTD; Tokyo, Japan) was carried out for patients by two ophthalmologists to detect diabetic retinopathy. It was preceded by visual acuity examination and pupillary dilatation. 
Ultrasound Doppler (Sonoline Elegra, Siemens Medical Systems, Inc., Ultrasound Group; Issaquah, WA, USA) of the carotid arteries was carried out for 41 out of 57 patients $(71.9 \%)$ to detect peripheral vascular disease. The latter was considered, if the lumen stenosis was $\geq 40 \%$.

Biochemical tests: The HbA1c (Glycated hemoglobin) was measured in the central laboratory of Al-Imamein Al-Kadhimein Medical City, and lipid profile including serum total cholesterol, serum triglycerides, serum HDL, serum LDL and VLDL was measured in the laboratories of Al-Nahrain College of Medicine.

\section{Statistical analysis}

The statistical analysis was performed with SPSS for Windows software v.10. Results are expressed as means \pm SD or $n(\%)$, and $P$ values $<0.05$ were considered to indicate significance.
Comparisons of data were performed using Student's t test and Fisher's exact test for continuous and categorical data, respectively. Odd ratios (OR) of relative risk were calculated and the results were given as OR and 95\% confidence interval $(\mathrm{Cl})$. OR expressed the increased risk of SMI per unit increase of the atherogenic risk factor itself. Multivariate analysis by multiple logistic regression ${ }^{105}$ was computed for the determination of independent risk factors for CAD.

\section{Results}

Sixty-three type 2 diabetic patients (group I \& II) were recruited. They comprised 37 male and 26 female patients aged 35 to 69 year with duration of DM ranging from 2 months to 24 years having characteristic features shown in table 1.

Table 1. Characteristics of the patients in terms of demographic data and metabolism

\begin{tabular}{cc}
\hline Parameters & Results \\
Number & 63 \\
Male/female & $37 / 26$ \\
Age (years) & $52.9 \pm 8.9$ \\
Age of onset of DM (years) & $46.3 \pm 8.7$ \\
Duration of DM (years) & $6.2 \pm 6.6$ \\
HbA1c (\%) & $8.8 \pm 1.8$ \\
Cholesterol (mmol/L) & $5.4 \pm 1.4$ \\
LDL cholesterol (mmol/L) & $3.4 \pm 1.4$ \\
HDL cholesterol (mmol/L) & $1.1 \pm 0.4$ \\
Triglyceride (mmol/L) & $1.8 \pm 0.6$ \\
Hyperlipidemia [n (\%)] & $29(46.0)$ \\
Family history of CAD [n (\%)] & $20(31.7)$ \\
Hypertension [n (\%)] & $31(49.2)$ \\
Smoking [n (\%)] & $19(30.1)$ \\
PVD [n (\%)] & $3(4.8)$ \\
Retinopathy [n (\%)] & $9(14.3 \%)$ \\
\hline Treatment & \\
Diet [n (\%)] & $4(6.3)$ \\
OHA (Daonil) [n (\%)] & $47(74.6)$ \\
Insulin / insulin + OHA [n (\%)] & $12(19.1)$ \\
Antihypertensive agent [n (\%)] & $24(38.1)$ \\
Aspirin [n (\%)] & $5(7.9)$ \\
\hline
\end{tabular}


The results of EST were non-diagnostic (nonconclusive) in 6 patients (as shown in patients' flow chart) (Figure 1): 2 because of fatigue, 2 because of hypertensive response, 1 because of dizziness, and 1 because of poor compliance. These conditions resulted in early termination of EST before reaching the target heart rate. Those patients were excluded from the study. The results of EST were diagnostic in the remaining 57 patients ( 22 from group I, and 35 from group (I). These patients were divided into those having a positive EST $(n=11,19.3 \%)$ and those having a negative EST $(n=46,80.7 \%)$. The positive EST results were more in group II than in group I. Of group II ( $n=35,61.4 \%), 10$ patients (28.6\%) had positive EST and 25 patients $(71.4 \%)$ had negative EST. While in group I ( $n=22,38.6 \%), 1$ patient (4.5\%) had positive EST and 21 patients (95.5\%) had negative EST (Figure 1, Table 2).

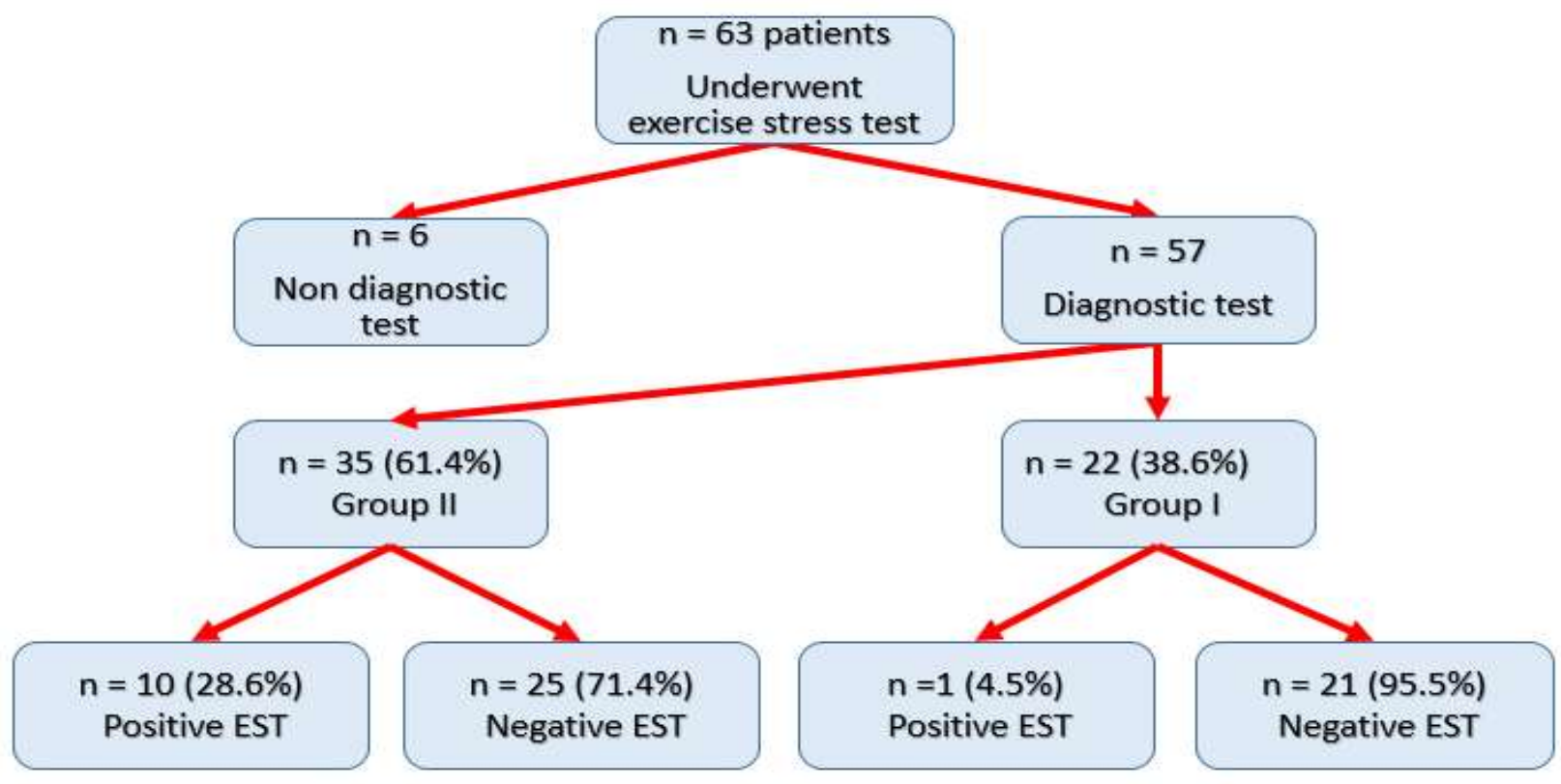

Figure 1. Patients' flow chart

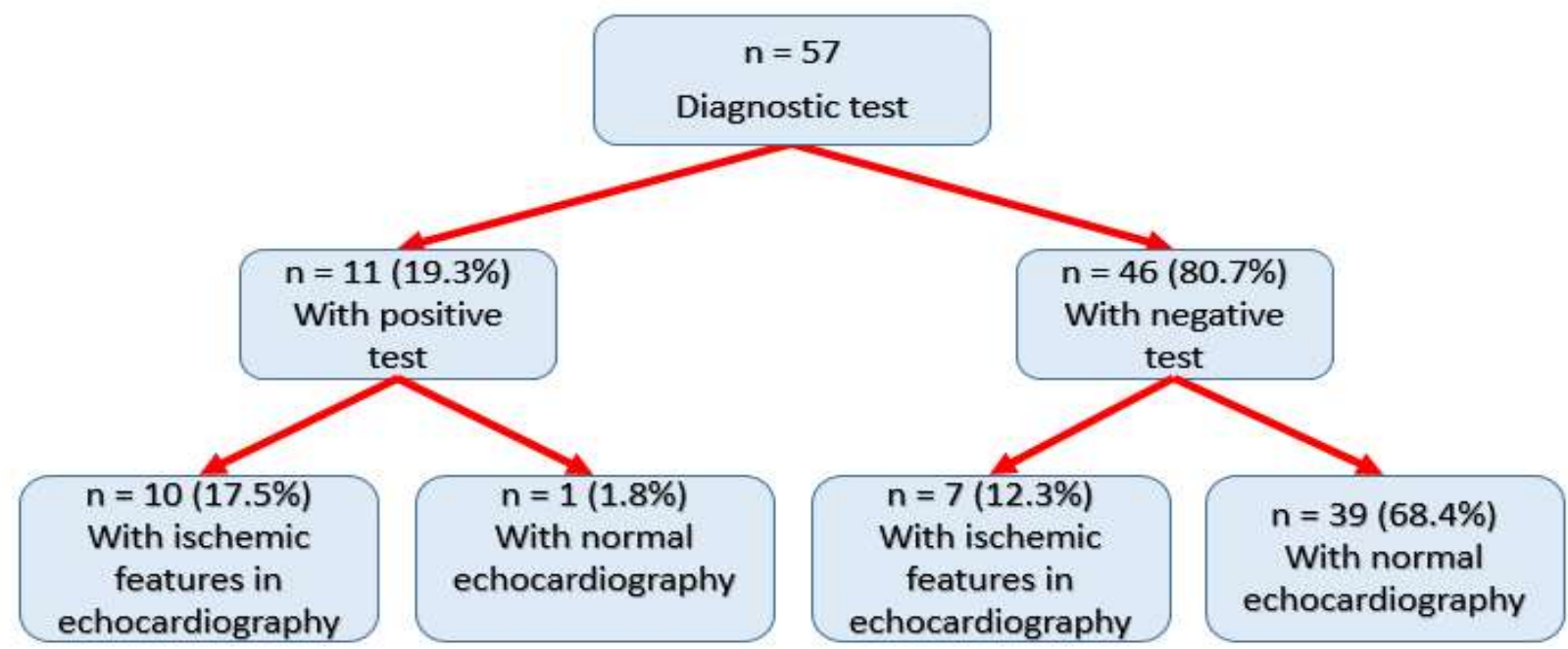


Figure 2. Echocardiographic results in $\mathbf{5 7}$ type 2 diabetic patients regarding their positive and negative results in exercise stress test

Table 2. Positive and negative patient clinical and metabolic data at exercise stress test

\begin{tabular}{cccc}
\hline Clinical and metabolic data & $\begin{array}{c}\text { Positive } \\
\mathrm{N}=11\end{array}$ & $\begin{array}{c}\text { Negative } \\
\mathrm{N}=46\end{array}$ & P value \\
\hline Male/female & $6 / 5$ & $27 / 19$ & 1.000 \\
Age (years) & $53.8 \pm 8.9$ & $53.1 \pm 9.0$ & 0.817 \\
Age of onset of DM (years) & $47.6 \pm 8.6$ & $46.0 \pm 9.2$ & 0.602 \\
Duration of DM (years) & $5.7 \pm 5.2$ & $6.7 \pm 6.1$ & 0.618 \\
HbA1c (\%) & $8.7 \pm 2.3$ & $8.9 \pm 1.8$ & 0.755 \\
Cholesterol (mmol/L) & $5.6 \pm 1.3$ & $5.4 \pm 1.4$ & 0.668 \\
LDL cholesterol (mmol/L) & $3.7 \pm 1.4$ & $3.4 \pm 1.5$ & 0.549 \\
HDL cholesterol (mmol/L) & $1.1 \pm 0.6$ & $1.2 \pm 0.3$ & 0.427 \\
Triglyceride (mmol/L) & $1.9 \pm 0.6$ & $1.8 \pm 0.6$ & 0.621 \\
Hyperlipidemia [n (\%)] & $9(81.8)$ & $17(37)$ & $0.016^{*}$ \\
Family history [n (\%)] & $5(45.5)$ & $15(32.6)$ & 0.491 \\
Hypertension [n (\%)] & $9(81.8)$ & $20(43.5)$ & $0.041^{*}$ \\
Smokers [n (\%)] & $5(45.5)$ & $12(26.1)$ & 0.275 \\
PVD [n (\%)] & $2(18.2)$ & $1(2.2)$ & 0.092 \\
Retinopathy [n (\%)] & $1(9.1)$ & $8(17.4)$ & 0.673 \\
\hline Treatments & & & \\
Diet & $1(9.1)$ & $3(6.5)$ & 1.000 \\
OHA (Daonil) & $8(72.7)$ & $33(71.8)$ & 1.000 \\
Insulin/insulin + OHA & $2(18.2)$ & $10(21.7)$ & 1.000 \\
Antihypertensive agent & $6(54.5)$ & $16(34.8)$ & 0.305 \\
Aspirin & $1(9.1)$ & $4(8.7)$ & 1.000 \\
\hline
\end{tabular}

Unpaired ttest for comparison of means, Fisher exact test for comparison of frequencies, * significant

The positive EST results were also more in patients with three atherogenic risk factors. Of the total number of patients with positive EST $(n=11,19.3 \%), 8$ patients $(14 \%)$ had three risk factors, 2 patients (3.5\%) had two risk factors, and only 1 patient (1.7\%) had one risk factor. The positive EST was diagnosed by a horizontal ST segment depression of $\geq 1 \mathrm{~mm}(0.1 \mathrm{mV})$ calculated at $0.08 \mathrm{~s}$ after the J point between QRS and ST segment None of the patients with positive EST had chest pain or down sloping or slow upsloping ST segment depression. Of the 11 patients with positive EST, 10 patients (17.5\%) (from group II) had one or more of the ischemic features in echocardiography (hypokinesia, dyskinesia, poor left ventricular function) and in the remaining patient (1.8\%) (from group I) the echocardiography was normal. In the 46 patients with negative EST, 7 patients (12.3\%) (5 patients from group II \& 2 patient from group I) showed abnormal echocardiography with ischemic features, while the remaining 39 patients (68.4\%) (20 patients from group II \& 19 from group I) the echocardiography was normal (Fig. 2). All patients had good LV functions with ejection fraction $>60 \%$. Three patients had infrequent ectopics. None of the patients had other abnormality in echocardiography like valvular heart disease or arrhythmias. Echocardiography was interpreted by two independent specialized persons in echocardiography. Ultrasound Doppler was carried out for 41 patients (65.1\%). Of the 11 patients with positive EST, 2 patients $(18.2 \%)$ (from group II) had carotids stenosis $>40 \%$ of the lumen, and 6 patients (54.5\%) (from group II) had normal Doppler study. The test was not 
done for the remaining 3 patients with positive EST. While 1 patient (2.2\%) (from group II) with negative EST had carotids stenosis $>40 \%$ of the lumen, and 32 patients (78.1\%) (20 patients from group II \& 12 patients from group I) had normal carotids. Diabetic retinopathy was observed by fundoscopy in 9 patients (14.3\%): Only one patient (9.1\%) (from group II) with diabetic retinopathy had positive EST. The remaining 8 patients (17.4\%) (6 patients from group II and 2 patients from group I) had negative EST. The fundoscopy was done by two ophthalmologists. In 26 patients (41.3\%) who had abnormal lipid profile test (hyperlipidemic patients), 9 patients (81.8\%) (all of them from group II) had positive EST. While the other 17 hyperlipidemic patients (37\%) (14 patients from group II \& 3 patients from group I) had negative EST. HbA1c was elevated above normal levels (i.e., $>6.5 \%$ ) in 50 patients (79.4).
9 (81.1) out of 11 patients with positive EST had elevated HbA1c $(9.2 \pm 2.4 \%)$. While 41 (89.1) out of 46 patients with negative EST had

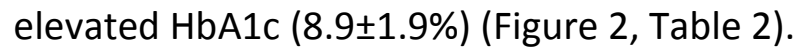

A multiple logistic regression analysis (multivariate analysis) was performed with silent myocardial ischemia as a dependent variable and the following atherogenic risk factors as predictive variables: PVD, family history of myocardial ischemia, smoking, hypertension, and hyperlipidemia. Analysis showed that PVD (OR=10.9, 95\% Cl=1.9-48.4; $\mathrm{P}=0.010$ ), hyperlipidemia (OR=9.3, 95\% $\mathrm{Cl}=1.8$ 37.1; $\mathrm{P}=0.012$ ), smoking ( $\mathrm{OR}=2.4,95 \% \mathrm{Cl}=1.6-$ 9.4; $\mathrm{P}=0.020)$, and hypertension ( $\mathrm{OR}=5.6,95 \%$ $\mathrm{Cl}=1.1-28.8 ; \quad \mathrm{P}=0.048) \quad$ were significant predictors of silent myocardial ischemia in type II diabetes. The role of family history of myocardial ischemia was non-significant (OR=1.9, 95\% Cl=0.5-7.3; 0.740) (Table 3).

Table 3. Multivariate analysis was performed with silent myocardial ischemia as a dependent variable and the following atherogenic risk factors as predictive variables

\begin{tabular}{cccc}
\hline Clinical and metabolic data & OR & $\mathbf{9 5 \%} \mathbf{C l}$ & $\mathbf{P}$ \\
\hline Family history & 1.9 & $0.5-7.3$ & 0.74 \\
Hyperlipidemia & 9.3 & $1.8-37.1$ & $0.012^{*}$ \\
Hypertension & 5.6 & $1.1-28.8$ & $0.048^{*}$ \\
Smoking & 2.4 & $1.6-9.4$ & $0.02^{*}$ \\
PVD & 10.9 & $1.9-48.4$ & $0.01^{*}$ \\
\hline
\end{tabular}

\footnotetext{
* significant
}

Risk of SMI in diabetic female patients slightly exceeded that of male patients, but with no significant difference. Their results were $(1.9$, 95\% $\mathrm{Cl}=0.5-6.9 ; \mathrm{P}=0.521)$ and $(\mathrm{OR}=0.8,95 \%$
$\mathrm{Cl}=0.2-2.9 ; \mathrm{P}=0.628$ ) respectively. No relation was found between retinopathy and SMI in both groups of patients (OR=0.2, 95\% $\mathrm{Cl}=0.02$ 1.8; $P=0.321$ ) (Table 4).

Table 4. Risk of SMI according to gender and retinopathy according to Fisher's exact test

\begin{tabular}{cccc}
\hline Clinical data & OR & $\mathbf{9 5 \%} \mathbf{~ C l}$ & $\mathbf{P}$ \\
\hline Males & 0.8 & $0.2-2.9$ & 0.628 \\
Females & 1.9 & $0.5-6.9$ & 0.521 \\
Retinopathy & 0.2 & $0.02-1.8$ & 0.321 \\
\hline
\end{tabular}




\section{Discussion}

Detection of subclinical CAD in diabetic patients is becoming more focused as diabetes becomes more common and additional information and tools to reduce the risk of death from CAD are becoming more available (5). Epidemiological studies clearly show the independent major impact to diabetes CAD, but also show cumulative risk rise of other atherogenic factors ${ }^{(6)}$. A higher mortality risk significantly associates with ST segment depression during EST, regardless of the presence or absence of angina (7). Mortality among patients with SMI is higher in diabetics than in non-diabetic subjects. Furthermore, the age-adjusted annual cardiovascular mortality rate is higher in type 2 diabetic patients with 2 or 3 cardiovascular risk factors than those with less than 2 risk factors, being $1.2-5.4 \%$ and $0.3-$ $0.6 \%{ }^{(8)}$, respectively. Taken together, our data strongly indicates that SMI should be screened for those patients with type 2 DM that have additional CAD risk factors.

This study has evaluated two groups of type 2 diabetic patients without any clinical or electrocardiographic evidence of CAD to detect SMI among these patients. Diabetic patients comprising group 2 (with 2 or more atherogenic risk factors) had showed more positive EST than those in group I (with 1 or no risk factor), $17.5 \%$ and $1.8 \%$, respectively. This is in agreement with other studies (9) and support the idea that only patients with 2 or more atherogenic risk factors should be screened with treadmill for SMI, otherwise it would be costly, exhausted for time and effort, and impracticable ${ }^{(10)}$. The traditional (basic) atherogenic risk factors were considered. These are peripheral vascular disease, family history of myocardial ischemia, smoking, hypertension, and hyperlipidemia. There are other risk factors revealed to be associated with SMI or CAD in some studies, were not considered, except for retinopathy and hyperglycemia (HbA1c), for example: microalbuminuria ${ }^{(11)}$, autonomic neuropathy (12), and apolipoprotein (a) ${ }^{(13)}$. This study had showed an interesting finding, that is: as the number of the basic risk factors increases, the results of positive EST (which most likely suggests the occurrence of SMI) increases in type 2 diabetic patients.

In this study, the percentage of SMI in type 2 diabetic patients was $19.3 \%$, SMI prevalence data reported in diabetic patients are quite variable from $9 \%$ to $48 \%{ }^{(14)}$. This variability may be due to the selection parameters and diagnostic approaches for the different patients, e.g. age, type and duration of DM.

Based on the results of multivariate analysis, PVD, hyperlipidemia, smoking, and hypertension appear to be risk factors that play a role in identification of these patients. No significant role of family history of myocardial ischemia was found. This study revealed no significant difference in SMI between men and women, and this finding is consistent with the assumption that diabetic women lose their inherited protection against CAD (15). Furthermore, no differences were found in the detection of SMI in relation to age of the patient, duration of DM, age of onset of DM, hyperglycemia (HbA1c), and retinopathy.

The non-significant role of age could be attributed to the absence of restriction criteria for selection of age of the patients, where young and elderly patients were included in this study.

Diabetic patients selected in our study had various duration of DM (2 months- 26 years). For this reason, probably, the duration of DM showed no significant correlation with SMI as a risk factor.

Uncontrolled hyperglycemia (which can be best assessed by periodic measurement of $\mathrm{HbA} 1 \mathrm{c}$ (16) was reported as risk factor for CAD and poor outcome in some studies. Retinopathy is not a risk factor for SMI, as in other previous studies (17). Retinopathy was of very low frequency in patients with positive EST, as well as patients with negative EST. This might explain the insignificance of retinopathy as a risk factor for SMI by the multivariate analysis of the data. Moreover, it explains also as is a microvascular not macro vascular complication of DM (18). 
This study concluded that diabetic patients with atherogenic risk factors have a higher risk for SMI than those without risk factors and as the number of the atherogenic risk factors increases, the risk for SMI increases. EST can be considered as a practicable screening test for SMI in type 2 diabetic patients with 2 or more atherogenic risk factors and echocardiography can play a role in supporting the results of EST, when coronary angiography is not available. Therefore, EST is recommended in asymptomatic type II diabetic patients with 2 or more atherogenic risk factors, unless contraindicated.

\section{Acknowledgement}

Cordial thanks and deep gratitude to my supervisor Prof. Dr. Faik H. Mohammed for his great support, encouragements and precious counsel. It is a great pleasure to express my appreciation and gratitude to Prof. Dr. Hashim M. Al-Kadhimi in the Diabetic Clinic in AlImamein Al-Kadhimein Medical City for his great help and advices. I am profoundly grateful to the medical staff of the Treadmill Unit in Al-Imamein Al-Kadhimein Medical City especially Dr. Nazar J. Al-Jalabi for the great help, patience, and guidance.

\section{Author contribution}

Dr. Al-Ethary: substantial contributions to acquisition and interpretation of data, writing the manuscript and revising it critically for important intellectual content, performing the calculations and statistical analysis. Dr. Alrikabi: selection of patients and interpretation of EST results.

\section{Conflict of interest}

Authors declare no conflict of interest.

\section{Funding}

Self-funded.

\section{References}

1. Singleton MJ, German CA, Bertoni $A G$, et al. Association of silent myocardial infarction with major cardiovascular events in diabetes: The ACCORD trial. Diabetes Care. 2020; 43(4): e45-e46. doi: 10.2337/dc19-2201.
2. Lovic D, Piperidou A, Zografou I, et al. The growing epidemic of diabetes mellitus. Curr Vasc Pharmacol. 2020; 18(2): 104-9. doi: $10.2174 / 1570161117666190405165911$.

3. Jacqueminet $S$, Barthélémy $O$, Le Feuvre $C$. Screening of silent myocardial ischemia in type 2 diabetic patients: a randomized trial comparing isotopic and echocardiographic stress tests. Diabetes Care. 2010; 33(6): e79. doi: 10.2337/dc09-2248.

4. Raggi P. Screening for atherosclerotic cardiovascular disease in patients with type 2 diabetes mellitus: controversies and guidelines. Can J Diabetes. 2020; 44(1): 86-92. doi: 10.1016/j.jcjd.2019.08.009.

5. Bertoluci MC, Rocha VZ. Cardiovascular risk assessment in patients with diabetes. Diabetol Metab Syndr. 2017; 9: 25. doi: 10.1186/s13098-017-0225-1.

6. Qin Z, Zhou K, Li Y, et al. The atherogenic index of plasma plays an important role in predicting the prognosis of type 2 diabetic subjects undergoing percutaneous coronary intervention: results from an observational cohort study in China. Cardiovasc Diabetol. 2020; 19(1): 23. doi: 10.1186/s12933-0200989-8.

7. Deng $X Q, X u X J, W u S H$, et al. Association between resting painless ST-segment depression with sudden cardiac death in middle-aged population: A prospective cohort study. Int J Cardiol. 2020; 301: 16. doi: 10.1016/j.ijcard.2019.11.148.

8. Mohan V, Venkatraman JV, Pradeepa R. Epidemiology of cardiovascular disease in type 2 diabetes: the Indian scenario. J Diabetes Sci Technol. 2010; 4(1): 158-70. doi: 10.1177/193229681000400121.

9. Albers AR, Krichavsky MZ, Balady GJ. Stress testing in patients with diabetes mellitus: diagnostic and prognostic value. Circulation. 2006; 113(4): 583-92. doi: 10.1161/CIRCULATIONAHA.105.584524.

10. Kim MK, Baek KH, Song KH, Kwon HS, Lee JM, Kang MI, Yoon KH, Cha BY, Son HY, Lee KW. Exercise treadmill test in detecting asymptomatic coronary artery disease in type 2 diabetes mellitus. Diabetes Metab J. 2011; 35(1): 34-40. doi: 10.4093/dmj.2011.35.1.34.

11. Hussain $Q$, Bukhari $M H, A f z a a l ~ F$, et al. Microalbuminuria (moderate albumin excretion) and its relationship with Silent myocardial ischemia in treatment naïve type II diabetic patients. Pak J Med Sci. 2020; 36(3): 496-500. doi: 10.12669/pjms.36.3.938.

12. Massardo T, Araya AV, Prat $H$, et al. Factors associated with silent myocardial ischemia, autonomic or peripheral neuropathies, and survival in diabetes mellitus type 2 patients without cardiovascular symptoms. Int J Diabetes Dev Ctries. 2020; 40: 80-6. doi: 10.1007/s13410-019-00758-7.

13. Richardson TG, Sanderson E, Palmer TM, et al. Evaluating the relationship between circulating lipoprotein lipids and apolipoproteins with risk of coronary heart disease: A multivariable Mendelian 
randomisation analysis. PLoS Med. 2020; 17(3): e1003062. doi: 10.1371/journal.pmed.1003062.

14. Zellweger MJ, Hachamovitch $R$, Kang $X$, et al. Prognostic relevance of symptoms versus objective evidence of coronary artery disease in diabetic patients. Eur Heart J. 2004; 25(7): 543-50. doi: 10.1016/j.ehj.2004.02.013.

15. Madonna R, Balistreri CR, De Rosa $S$, et al. Impact of sex differences and diabetes on coronary atherosclerosis and ischemic heart disease. J Clin Med. 2019; 8(1): 98. doi: 10.3390/jcm8010098.

16. Sherwani SI, Khan HA, Ekhzaimy A, et al. Significance of $\mathrm{HbA1C}$ test in diagnosis and prognosis of diabetic patients. Biomark Insights. 2016; 11: 95-104. doi: 10.4137/BMI.S38440.

17. El-Hefny E, Tag El-Din A, Sadek A, et al. The Degree of Retinopathy Correlates with the Presence of Silent
Myocardial Ischemia in Diabetic Patients. Cardiol Cardiovasc Res. 2020; 4(2): 34. doi: 10.11648/j.ccr.20200402.12.

18. Turner RC, Millns $\mathrm{H}$, Neil HAW, et al. Risk factors for coronary artery disease in non-insulin dependent diabetes mellitus: United Kingdom Prospective Diabetes Study (UKPDS: 23). BMJ. 1998; 316(7134): 823-8. doi: $10.1136 / \mathrm{bmj} .316 .7134 .823$.

\footnotetext{
Correspondence to Dr. Zeki Y. Al-Ethary E-mail: zeki_yahya@yahoo.com

Received Sep. $2^{\text {nd }} 2020$

Accepted Nov. $15^{\text {th }} 2020$
} 